\title{
Coronagraphic phase diversity: performance study and laboratory demonstration
}

\author{
B. Paul $1^{1,2,3}$ J.-F. Sauvage ${ }^{1,3}$, and L. M. Mugnier ${ }^{1,3}$ \\ 1 Onera - The French Aerospace Lab, 92322 Chatillon, France \\ e-mail: baptiste.paul@onera.fr \\ 2 Aix Marseille Université, CNRS, LAM (Laboratoire d'Astrophysique de Marseille) UMR 7326, 13388 Marseille, France \\ ${ }^{3}$ Groupement d'intérêt scientifique PHASE (Partenariat Haute résolution Angulaire Sol et Espace) between Onera, Observatoire \\ de Paris, CNRS, Université Diderot, Laboratoire d'Astrophysique de Marseille and Institut de Planétologie et d'Astrophysique de \\ Grenoble, France
}

Received 17 December 2012 / Accepted 31 January 2013

\begin{abstract}
Context. The final performance of current and future instruments dedicated to exoplanet detection and characterization (such as SPHERE on the European Very Large Telescope, GPI on Gemini North, or future instruments on Extremely Large Telescopes) is limited by uncorrected quasi-static aberrations. These aberrations create long-lived speckles in the scientific image plane, which can easily be mistaken for planets.

Aims. Common adaptive optics systems require dedicated components to perform wave-front analysis. The ultimate wave-front measurement performance is thus limited by the unavoidable differential aberrations between the wave-front sensor and the scientific camera. To reach the level of detectivity required by high-contrast imaging, these differential aberrations must be estimated and compensated for. In this paper, we characterize and experimentally validate a wave-front sensing method that relies on focal-plane data. Methods. Our method, called COFFEE (for COronagraphic Focal-plane wave-Front Estimation for Exoplanet detection), is based on a Bayesian approach, and it consists in an extension of phase diversity to high-contrast imaging. It estimates the differential aberrations using only two focal-plane coronagraphic images recorded from the scientific camera itself.

Results. We first present a thorough characterization of COFFEE's performance by means of numerical simulations. This characterization is then compared with an experimental validation of COFFEE using an in-house adaptive optics bench and an apodized Roddier \& Roddier phase mask coronagraph. An excellent match between experimental results and the theoretical study is found. Lastly, we present a preliminary validation of COFFEE's ability to compensate for the aberrations upstream of a coronagraph.
\end{abstract}

Key words. instrumentation: adaptive optics - instrumentation: high angular resolution - techniques: image processing methods: numerical - methods: laboratory - telescopes

\section{Introduction}

Exoplanet imaging is one of the main challenges in today's astronomy. A direct observation of these planets can provide information on both the chemical composition of their atmospheres and their temperatures. Such observations have recently been made possible (Kalas et al. 2008; Marois et al. 2008; Lagrange et al. 2009), but only thanks to their high mass or their wide apparent distance from their host star.

Being able to image an object as faint as an extra-solar planet very close to its parent star requires the use of extreme AO (XAO) systems coupled to a high-contrast imaging technique, such as coronagraphy. Instruments dedicated to exoplanet imaging using these two techniques (SPHERE on the VLT, Beuzit et al. 2007; GPI on Gemini North, Macintosh et al. 2008) are currently being integrated. The performance of such systems is limited by residual speckles on the detector. These speckles, which originate in quasi-static non common path aberrations (NCPA), strongly decrease the extinction provided by the coronagraph and can be difficult to distinguish from an exoplanet. To achieve the ultimate system performance, these aberrations must be measured and compensated for. The currentgeneration instruments, SPHERE and GPI, respectively rely on phase diversity (Gonsalvez 1982) and an interferometry approach (Wallace et al. 2010) to compensate for these NCPA.

Several techniques dedicated to high-contrast imaging system optimization have been proposed for future systems. Some of them rely on a dedicated wave-front sensing hardware (Guyon et al. 2009), others use scientific focal plane data assuming small aberrations. Speckle nulling iterative techniques (Bordé \& Traub 2006; Give'on et al. 2007) estimate the electric field in the detector plane using at least three images. The technique proposed by Baudoz et al. (2006) relies on a modification of the imaging system, but requires only one image. These techniques aim at minimizing the energy in a chosen area ("dark hole"), leading to a contrast optimization on the detector (Trauger et al. 2010; Baudoz et al. 2012) in a closed loop process.

We have recently proposed a focal-plane wave-front sensor, COFFEE (Sauvage et al. 2012), which is an extension of conventional phase diversity (Mugnier et al. 2006) to a coronagraphic system. Since COFFEE uses focal-plane images, it is possible to characterize the whole bench without any differential aberration. This method requires only two focal-plane images to estimate the aberrations upstream of the coronagraph without any modification of the coronagraphic imaging system or assuming small aberrations. COFFEE's principle and its application to the 


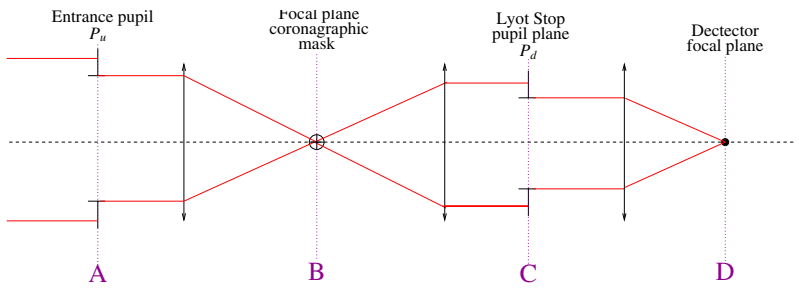

Fig. 1. Coronagraphic imaging instrument: principle.

apodized Roddier \& Roddier phase mask (ARPM) are described in Sect. 2. In Sect. 3, we evaluate the quality of NCPA estimation by realistic simulations. In Sect. 4, we present the experimental results from the laboratory demonstration of COFFEE on an inhouse adaptive optics bench (BOA) with an ARPM. Section 5 concludes the paper.

\section{COFFEE: principle}

\subsection{Extension of phase diversity to coronagraphic images}

Figure 1 describes the coronagraphic imaging scheme considered in this paper. We consider four successive planes denoted A (circular entrance pupil of diameter $D_{\mathrm{u}}$ ), B (coronagraphic focal plane), C (Lyot Stop), and D (detector plane). The optical aberrations are considered as static and introduced in the pupil planes A and C. The coronagraphic device is composed of a focal plane mask located in plane B and a Lyot Stop situated in plane C. No particular assumption is made on the pupil shape or intensity. Thus, the description of COFFEE is compatible with several coronagraphic devices. COFFEE uses two images, $i_{\mathrm{c}}^{\mathrm{f}}$ and $\boldsymbol{i}_{\mathrm{c}}^{\mathrm{d}}$, recorded on the detector (plane D in Fig. 1) that, as in phase diversity, differ from a known aberration, $\phi_{\text {div }}$, to estimate aberrations both upstream $\left(\boldsymbol{\phi}_{\mathrm{u}}\right)$ and downstream $\left(\boldsymbol{\phi}_{\mathrm{d}}\right)$ of the coronagraph.

Considering the calibration of the instrument with an unresolved object, we use the following imaging model:

$$
\begin{aligned}
& \boldsymbol{i}_{\mathrm{c}}^{\text {foc }}=\alpha \cdot \boldsymbol{h}_{\mathrm{det}} \star \boldsymbol{h}_{\mathrm{c}}\left(\boldsymbol{\phi}_{\mathrm{u}}, \boldsymbol{\phi}_{\mathrm{d}}\right)+\boldsymbol{n}^{\mathrm{foc}}+\beta \\
& \boldsymbol{i}_{\mathrm{c}}^{\mathrm{div}}=\alpha \cdot \boldsymbol{h}_{\mathrm{det}} \star \boldsymbol{h}_{\mathrm{c}}\left(\boldsymbol{\phi}_{\mathrm{u}}+\boldsymbol{\phi}_{\mathrm{div}}, \boldsymbol{\phi}_{\mathrm{d}}\right)+\boldsymbol{n}^{\mathrm{div}}+\beta
\end{aligned}
$$

where $\alpha$ is the incoming flux, $\boldsymbol{h}_{\mathrm{c}}$ the coronagraphic "point spread function" (PSF) of the instrument (i.e. the response of a coronagraphic imaging system to a point source), $\boldsymbol{h}_{\mathrm{det}}$ the known detector PSF, $\boldsymbol{n}^{\text {foc }}$ and $\boldsymbol{n}^{\text {div }}$ are the measurement noises, $\beta$ is a uniform background (offset), and $\star$ denotes the discrete convolution operation. Such an imaging model can be used for any coronagraphic PSF expression $\boldsymbol{h}_{\mathrm{c}}$. The measurement noises $\boldsymbol{n}^{\text {foc }}$ and $\boldsymbol{n}^{\mathrm{div}}$ comprise both photon and detector noises. Because calibration is assumed to be performed with high flux levels, we adopt a non-stationary white Gaussian model, which is a good approximation of a mix of photon and detector noises. Its variance is the sum of the photon and detector noise variances: $\sigma_{\mathrm{n}}^{2}[t]=\sigma_{\mathrm{ph}}^{2}[t]+\sigma_{\mathrm{det}}^{2}$ (Mugnier et al. 2004), with $t$ the pixel position in the detector plane. The former can be estimated as the image itself thresholded to positive values, and the latter can be calibrated prior to the observations.

We adopt a maximum a posteriori (MAP) approach and estimate the aberrations $\phi_{\mathrm{u}}$ and $\phi_{\mathrm{d}}$, the flux $\alpha$, and the background $\beta$ that minimize the neg-log-likelihood of the data, potentially penalized by regularization terms on $\phi_{\mathrm{u}}$ and $\phi_{\mathrm{d}}$ designed to enforce smoothness of the sought phases:

$$
\left(\hat{\alpha}, \hat{\beta}, \hat{\boldsymbol{\phi}}_{\mathrm{u}}, \hat{\boldsymbol{\phi}}_{\mathrm{d}}\right)=\underset{\alpha, \beta, \boldsymbol{\phi}_{\mathrm{u}}, \boldsymbol{\phi}_{\mathrm{d}}}{\arg \min } J\left(\alpha, \beta, \boldsymbol{\phi}_{\mathrm{u}}, \boldsymbol{\phi}_{\mathrm{d}}\right)
$$

where

$$
\begin{aligned}
J\left(\alpha, \beta, \boldsymbol{\phi}_{\mathrm{u}}, \boldsymbol{\phi}_{\mathrm{d}}\right)= & \frac{1}{2}\left\|\frac{\boldsymbol{i}_{\mathrm{c}}^{\mathrm{foc}}-\left(\alpha \cdot \boldsymbol{h}_{\mathrm{d}} \star \boldsymbol{h}_{\mathrm{c}}\left(\boldsymbol{\phi}_{\mathrm{u}}, \boldsymbol{\phi}_{\mathrm{d}}\right)+\beta\right)}{\boldsymbol{\sigma}_{\mathrm{n}}^{\mathrm{foc}}}\right\|^{2} \\
& +\frac{1}{2}\left\|\frac{\boldsymbol{i}_{\mathrm{c}}^{\mathrm{div}}-\left(\alpha \cdot \boldsymbol{h}_{\mathrm{d}} \star \boldsymbol{h}_{\mathrm{c}}\left(\boldsymbol{\phi}_{\mathrm{u}}+\boldsymbol{\phi}_{\mathrm{div}}, \boldsymbol{\phi}_{\mathrm{d}}\right)+\beta\right)}{\sigma_{\mathrm{n}}^{\mathrm{div}}}\right\|^{2} \\
& +\mathcal{R}\left(\boldsymbol{\phi}_{\mathrm{u}}\right)+\mathcal{R}\left(\boldsymbol{\phi}_{\mathrm{d}}\right)
\end{aligned}
$$

where $\|x\|^{2}$ denotes the sum of squared pixel values of map $\boldsymbol{x}$, $\sigma_{\mathrm{n}}^{\text {foc }}$, and $\sigma_{\mathrm{n}}^{\text {div }}$ are the noise standard deviation maps of each image, and $\mathcal{R}$ is a regularization metric for the phase.

Any aberration $\boldsymbol{\phi}$ is expanded on a basis $\left\{\boldsymbol{Z}_{k}\right\}$ that is typically either Zernike polynomials or the pixel indicator functions in the corresponding pupil plane: $\boldsymbol{\phi}=\sum_{k} a_{k} Z_{k}$ where the summation is, in practice, limited to the number of coefficients considered sufficient to correctly describe the aberrations. In this paper, the phase is expanded on a truncated Zernike basis. The impact of using a regularization metric with such a basis is studied later in this paper. In the MAP framework, the regularization metrics $\mathcal{R}\left(\phi_{\mathrm{u}}\right)$ and $\mathcal{R}\left(\phi_{\mathrm{d}}\right)$ are deduced from the assumed a priori statistics of $\phi_{\mathrm{u}}$ and $\boldsymbol{\phi}_{\mathrm{d}}$. Assuming these aberrations are zeromean, Gaussian, and neglecting a priori correlations between Zernike modes, we obtain, for an estimation performed on $N$ Zernike modes:

$\mathcal{R}\left(\boldsymbol{\phi}_{x}\right)=\frac{1}{2} \boldsymbol{a}_{x}^{t} R_{a_{x}}^{-1} \boldsymbol{a}_{x}=\frac{1}{2} \sum_{k=1}^{N} \frac{a_{x_{k}}^{2}}{\sigma_{x_{k}}^{2}}$,

where $\sigma_{x_{k}}^{2}$ is the assumed phase variance per Zernike mode, $R_{a_{k}}$ the covariance matrix, and $\boldsymbol{a}_{x}$ a $N$ element vector containing the estimated Zernike coefficients $a_{x_{k}}$. Here $x$ is either u (upstream) or d (downstream).

The minimization of metric $J\left(\alpha, \beta, \phi_{\mathrm{u}}, \phi_{\mathrm{d}}\right)$ of Eq. (3) is performed by means of a limited memory variable metric (BFGS) method (Press et al. 2007; Thiébaut 2002), which is a fast quasiNewton type minimization method. It uses both gradients $\frac{\partial J}{\partial \phi_{\mathrm{u}}}$ and $\frac{\partial J}{\partial \phi_{\mathrm{d}}}$. Flux $\alpha$ and offset $\beta$ are analytically obtained using gradients $\frac{\partial J}{\partial \alpha}$ and $\frac{\partial J}{\partial \beta}$ (implementation details, including gradient expressions, can be found in Appendix A).

Sauvage et al. (2012) established that a suitable diversity phase $\phi_{\text {div }}$ for COFFEE was a mix of defocus and astigmatism: $\boldsymbol{\phi}_{\text {div }}=a_{4}^{\text {div }} \boldsymbol{Z}_{4}+a_{5}^{\text {div }} \boldsymbol{Z}_{5}$ with $a_{4}^{\text {div }}=a_{5}^{\text {div }}=80 \mathrm{~nm}$ rms, introduced upstream of the coronagraph. We therefore use this diversity phase in the following.

\subsection{Coronagraphic imaging model}

The imaging model used by COFFEE in the criterion minimization (Eq. (3)) requires a coronagraphic PSF expression. In this paper, we use the analytical coronagraphic imaging model developed by Sauvage et al. (2010), whose formalism is developed in this section, where $\boldsymbol{r}$ is the pupil plane position vector, $r$ its modulus, and $\gamma$ the focal plane position vector. The entrance pupil function $\boldsymbol{P}_{\mathrm{u}}(\boldsymbol{r})$ is such that:

$\boldsymbol{P}_{\mathrm{u}}(\boldsymbol{r})=\boldsymbol{\Pi}\left(\frac{2 r}{D_{\mathrm{u}}}\right) \boldsymbol{\Phi}(\boldsymbol{r})$

with $\Pi\left(\frac{2 r}{D_{u}}\right)=1$ for $r \leq \frac{D_{u}}{2}$, pupil entrance diameter, 0 otherwise, and $\boldsymbol{\Phi}$ is an apodization function. In this paper, we consider that the impact of amplitude aberrations is negligible, which is a 
reasonable assumption for a ground-based, high-contrast imaging system such as SPHERE. Considering only static aberrations (no residual turbulent aberrations), the electric field $\boldsymbol{\Psi}_{\mathrm{A}}$ in the entrance pupil plane can be written as

$\boldsymbol{\Psi}_{\mathrm{A}}(\boldsymbol{r})=\boldsymbol{P}_{\mathrm{u}}(\boldsymbol{r}) \mathrm{e}^{j \phi_{\mathrm{u}}(\boldsymbol{r})}$.

The field amplitude $\boldsymbol{\Psi}_{\mathrm{B}}(\boldsymbol{\gamma})$ in plane B can be calculated, following Sauvage et al. (2010), using the analytical coronagraphic imaging model (which is called "perfect coronagraph model" hereafter):

$\boldsymbol{\Psi}_{\mathrm{B}}(\boldsymbol{\gamma})=\mathrm{FT}^{-1}\left(\boldsymbol{\Psi}_{\mathrm{A}}(\boldsymbol{r})\right)-\eta_{0} \mathrm{FT}^{-1}\left(\boldsymbol{P}_{\mathrm{u}}(\boldsymbol{r})\right)$,

where $\eta_{0}$ is the scalar that minimizes the outcoming energy from focal plane $\mathrm{B}$, whose analytical value is given by

$\eta_{0}=\frac{1}{\mathcal{N}} \iint_{\mathrm{S}} \boldsymbol{\Psi}_{\mathrm{A}}^{*}(\boldsymbol{r}) \boldsymbol{P}_{\mathrm{u}}(\boldsymbol{r}) \mathrm{d} \boldsymbol{r}$

where

$\mathcal{N}=\iint_{\mathrm{S}} \boldsymbol{P}_{\mathrm{u}}^{*}(\boldsymbol{r}) \boldsymbol{P}_{\mathrm{u}}(\boldsymbol{r}) \mathrm{d} \boldsymbol{r}$

It is worthy mentioning that $\eta_{0}$ is the exact definition of the instantaneous Strehl ratio given by Born \& Wolf (1989). One can notice that $\eta_{0}=1$ when there is no aberration upstream of the coronagraph $\left(\phi_{\mathrm{u}}(\boldsymbol{r})=0\right)$, so that $\boldsymbol{\Psi}_{\mathrm{B}}=0$ in such a case. No aberration in the entrance pupil leads to no outcoming energy from plane B, and thus to a perfect extinction in the detector plane $\mathrm{D}$.

Propagating the wave from plane B (Eq. (7)) to plane D, we can write the electric field $\boldsymbol{\Psi}_{\mathrm{D}}(\gamma)$ in the detector plane:

$$
\begin{array}{r}
\boldsymbol{\Psi}_{D}(\boldsymbol{\gamma})=\mathrm{FT}^{-1}\left\{\boldsymbol{P}_{\mathrm{d}}(\boldsymbol{r}) \mathrm{e}^{j\left(\boldsymbol{\phi}_{\mathrm{u}}(\boldsymbol{r})+\phi_{\mathrm{d}}(\boldsymbol{r})\right)}\right\} \\
-\eta_{0} \mathrm{FT}^{-1}\left\{\boldsymbol{P}_{\mathrm{d}}(\boldsymbol{r}) \mathrm{e}^{j \phi_{\mathrm{d}}(\boldsymbol{r})}\right\},
\end{array}
$$

where $\boldsymbol{P}_{\mathrm{d}}(\boldsymbol{r})$ is the Lyot stop pupil function: $\boldsymbol{P}_{\mathrm{d}}(\boldsymbol{r})=$ $\boldsymbol{\Pi}\left(\frac{2 r}{D_{\mathrm{d}}}\right) \boldsymbol{P}_{\mathrm{u}}(\boldsymbol{r})$, with $D_{d}$ the Lyot stop pupil diameter $\left(D_{\mathrm{d}} \leq D_{\mathrm{u}}\right)$. For the sake of simplicity, we omit the spatial variables $\boldsymbol{r}$ and $\gamma$ in the following. The coronagraphic PSF of the instrument, denoted by $\boldsymbol{h}_{\mathrm{c}}$, is the square modulus of $\boldsymbol{\Psi}_{\mathrm{D}}$ :

$$
\begin{aligned}
\boldsymbol{h}_{\mathrm{c}}\left(\boldsymbol{\phi}_{\mathrm{u}}, \boldsymbol{\phi}_{\mathrm{d}}\right)= & \mid \mathrm{FT}^{-1}\left(\boldsymbol{P}_{\mathrm{d}} \mathrm{e}^{j\left(\boldsymbol{\phi}_{\mathrm{u}}+\boldsymbol{\phi}_{\mathrm{d}}\right)}\right) \\
& -\left.\eta_{0} \mathrm{FT}^{-1}\left(\boldsymbol{P}_{\mathrm{d}} \mathrm{e}^{j \boldsymbol{\phi}_{\mathrm{d}}}\right)\right|^{2} .
\end{aligned}
$$

In this paper, this expression of the coronagraphic PSF is the one used by COFFEE for estimating $\phi_{\mathrm{u}}$ and $\boldsymbol{\phi}_{\mathrm{d}}$; i.e., Eq. (11) is inserted into the imaging model (Eq. (1)) used in the criterion minimization described in Eq. (3).

As described by Sauvage et al. (2010), this model, which analytically describes the impact of a coronagraph in an imaging system, considers that the coronagraph removes the projection of the incoming electric field on an Airy pattern, represented by the parameter $\eta_{0}$ (Eq. (8)). Since it does not assume small aberrations, it can be used for any wave-front error upstream of the coronagraph. The quality of the fit of this analytical imaging model with the ARPM coronagraph is discussed later in this

\begin{tabular}{|c|c|}
\hline \multicolumn{2}{|c|}{ Simulation } \\
\hline Image size & $\begin{array}{l}93 \times 93 \frac{\lambda}{D}(128 \times 128 \text { pixels, } \\
\text { oversampling factor: } 1.38)\end{array}$ \\
\hline Light spectrum & monochromatic $(\lambda=635 \mathrm{~nm})$ \\
\hline $\begin{array}{l}\text { Aberration upstream of the } \\
\text { coronagraph }\left(\phi_{\mathrm{u}}\right)\end{array}$ & $\mathrm{WFE}=80 \mathrm{~nm} \mathrm{rms}$ \\
\hline $\begin{array}{l}\text { Aberration downstream of } \\
\text { the coronagraph }\left(\phi_{\mathrm{d}}\right)\end{array}$ & $\mathrm{WFE}=20 \mathrm{~nm} \mathrm{rms}$ \\
\hline $\begin{array}{l}\text { Zernike basis used for } \phi_{\mathrm{u}} \\
\text { and } \phi_{\mathrm{d}} \text { simulation }\end{array}$ & 36 Zernike polynomials \\
\hline \multicolumn{2}{|c|}{ Phase estimation: COFFEE } \\
\hline $\begin{array}{l}\text { Zernike basis used for } \phi_{\mathrm{u}} \\
\text { and } \phi_{\mathrm{d}} \text { reconstruction }\end{array}$ & 36 Zernike polynomials \\
\hline Regularization metric & none \\
\hline
\end{tabular}
paper (Sect. 3.5).
Table 1. COFFEE: simulation parameters used for the performance assessments of Sects. 3.1-3.3.

\section{Performance assessment by numerical simulation}

The aim of this section is to quantify the impact of each error source on COFFEE's aberration estimation. Such a study will show COFFEE's sensitivity to the classical error sources that limit the phase retrieval in a real system (and thus the final extinction of the coronagraph), which will be of high interest in defining COFFEE's upgrades. Likewise, it will allow us to estimate the accuracy level expected on our AO bench. In this section, we present the evolution of this reconstruction error with respect to the incoming flux (Sect. 3.1), to the size of the source (Sect. 3.2), to an error made on the assumed diversity phase used in the reconstruction (Sect. 3.3), and to the number of Zernike modes used in the reconstruction (Sect. 3.4). For each error source, coronagraphic images will be computed using the imaging model presented in Eq. (1), using the perfect coronagraph model to calculate the coronagraphic PSF $h_{\mathrm{c}}$ whose expression is given Eq. (11). COFFEE will then perform the phase estimation using these two images. The compatibility of COFFEE with realistic coronagraphic images will be studied as well (Sect. 3.5) by computing coronagraphic images using a realistic coronagraph model and then running COFFEE to estimate the aberrations both upstream and downstream of the coronagraph.

Table 1 gathers the parameters used for these simulations.

The chosen wave-front error (WFE) values upstream and downstream of the coronagraph for these simulations are typical of the aberrations that will be estimated on our AO bench in Sect. 4 (so that experimental results can be compared to the following simulations). Since these simulations are performed with a small number of Zernike modes (36), there is no need of regularization metrics in such simulations.

To simulate realistic aberrations, we have considered that the variance per Zernike mode $\sigma_{k}^{2}$ was decreasing with the radial order $n(k)$ of the considered Zernike mode $k$ (Noll 1976):

$\sigma_{k}^{2} \propto \frac{1}{n(k)^{2}}$

This corresponds to a decrease in the static aberration spatial spectrum as $\frac{1}{|v|^{2}}$, where $v$ is the spatial frequency, which is a common assumption for mirror fabrication errors. To evaluate 

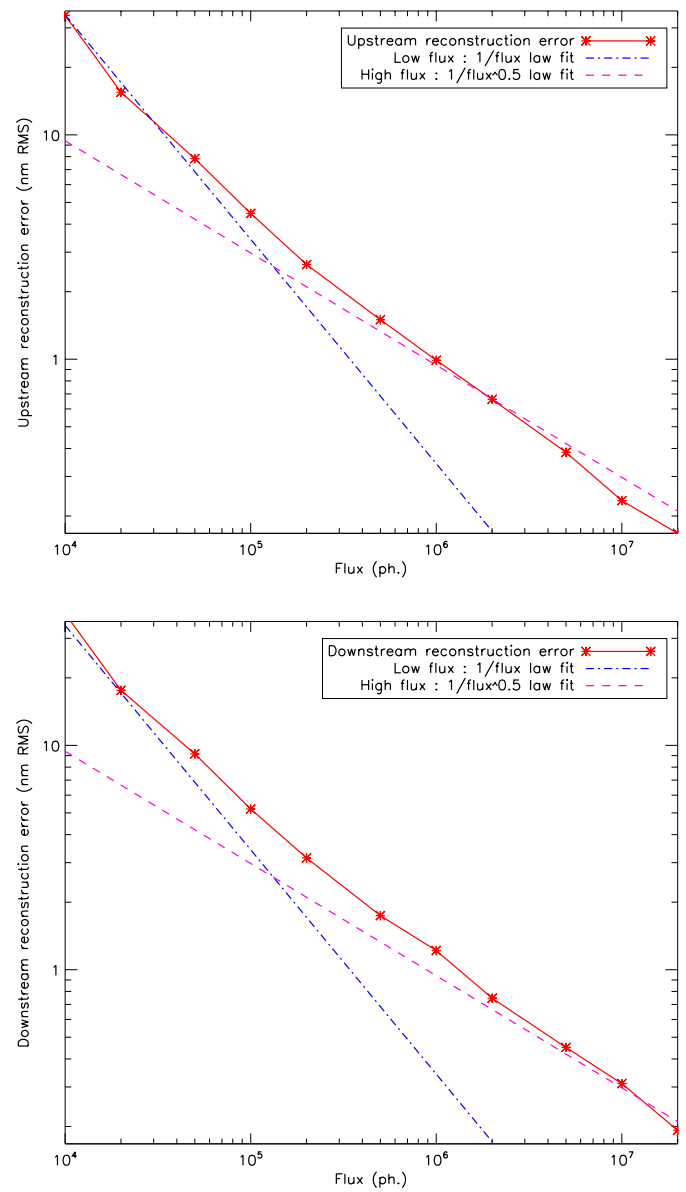

Fig. 2. Aberrations upstream $\left(\phi_{\mathrm{u}}(\mathrm{WFE}=80 \mathrm{~nm})\right.$, top $)$ and downstream $\left(\phi_{\mathrm{d}}(\mathrm{WFE}=20 \mathrm{~nm})\right.$, bottom $)$ of the coronagraph: reconstruction error (solid red line) as a function of the incoming flux $\alpha$. For comparison, $\frac{1}{\alpha}$ (cyan dashed line) and $\frac{1}{\sqrt{\alpha}}$ (magenta dashed line) theoretical behaviours are plotted for detector noise only and photon noise only (respectively).

COFFEE's performance, we define the reconstruction error $\epsilon_{x}$ ( $x$ stands for $\mathrm{u}$ (upstream) or $\mathrm{d}$ (downstream)) as

$\epsilon=\sqrt{\sum_{k=2}^{N-1}\left|a_{k}-\hat{a}_{k}\right|^{2}}$

with $a_{k}$ the Zernike coefficients (starting with $k=2$ corresponding to tilt) used for the simulation, $\hat{a}_{k}$ the reconstructed Zernike coefficients, and $N$ the number of Zernike modes. In this section, every reconstruction error value is an average value, computed from ten independent simulated phases.

\subsection{Noise propagation}

The ultimate limitation of an instrument lies in the amount of noise in the images. In Fig. 2, we present the reconstruction error for the aberrations upstream $\left(\boldsymbol{\phi}_{\mathrm{u}}\right)$ and downstream $\left(\boldsymbol{\phi}_{\mathrm{d}}\right)$ of the coronagraph with respect to the total incoming flux. Photon noise and detector noise $\left(\sigma_{\mathrm{det}}=6 \mathrm{e}^{-}\right)$are added in the coronagraphic images for simulation.

The evolution of the reconstruction error presented in Fig. 2 is proportional to $(1 / \alpha)$ for the detector noise limited regime (low flux) and to $(1 / \sqrt{\alpha})$ for the photon noise limited regime (high flux). In this figure, it can be seen that for an incoming

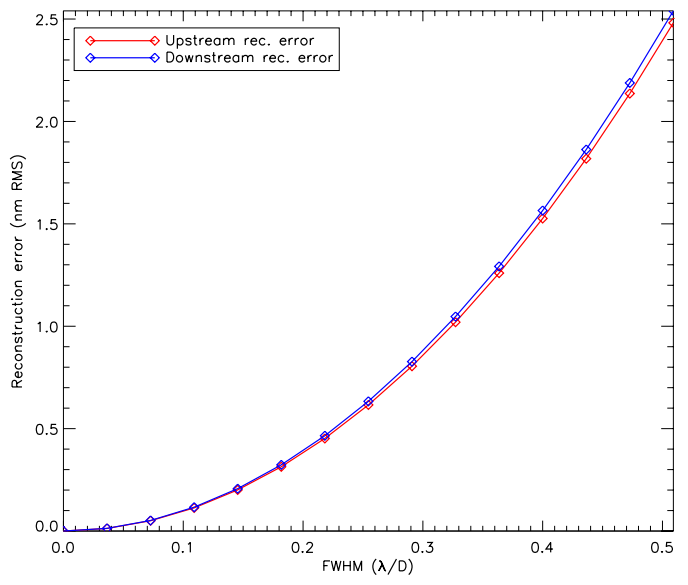

Fig. 3. Error reconstructions upstream (red line) and downstream (blue line) of the coronagraph as functions of the size of the source on the coronagraph.

flux $\alpha \geq 10^{6}$ photons, the reconstruction error $\epsilon_{\mathrm{u}}$ for the phase upstream of the coronagraph is smaller than $1 \mathrm{~nm}$ rms. Thus, in a calibration process, where high values of flux ( $\geq 10^{6}$ photons) can be easily reached, COFFEE's performance will not be significantly affected by noise.

It is noteworthy that the results of many similar simulations with various levels of upstream aberrations show that COFFEE's reconstruction error does not depend on the amplitude of the aberrations upstream of the coronagraph, as long as the diversity phase amplitude is larger than the WFE of the aberrations to be estimated.

\subsection{Impact of the source size on the reconstruction error}

Our imaging model, presented in Sect. 2.1 (Eq. (1)), assumes an unresolved object. Thus, the presence of a real source with a given spatial extension will have an impact on the phase reconstruction, which is quantified here. We consider here a Gaussianshaped laser source, emitted from a single-mode fiber. Because of the incoming light coherence, it can be represented as a Gaussian amplitude in the entrance pupil plane (where COFFEE assumes a uniform amplitude). Knowing this, coronagraphic images are simulated by considering a small coherent Gaussianshaped beam $\left(F W H M \leq 0.5 \frac{\lambda}{D}\right)$ on the coronagraph, and then processed by COFFEE.

Since the imaging model assumes an unresolved object, both reconstruction errors for the phases upstream and downstream of the coronagraph increase with the FWHM of the coherent object, as showed in Fig. 3, but remains low: for an FWHM smaller than $\frac{\lambda}{3 D}$, the reconstruction error is indeed sub-nanometric. The size of the laser source will thus definitely not be a limitation for COFFEE: if this error is not negligible in the total error budget, it is possible to include it in the imaging model used by COFFEE (Eq. (1)) as a non-uniform (Gaussian) entrance pupil function $\boldsymbol{P}_{\mathrm{u}}(\boldsymbol{r})$.

\subsection{Sensitivity to a diversity phase error}

The diversity phase $\boldsymbol{\phi}_{\text {div }}=a_{4}^{\text {div }} \boldsymbol{Z}_{4}+a_{5}^{\text {div }} \boldsymbol{Z}_{5}$ has been defined in Sect. 2.1. This phase $\phi_{\text {div }}$ is one of the inputs that COFFEE needs in order to perform phase retrieval, so it must be calibrated as accurately as possible. To optimize the use of COFFEE, the impact of an error on such a calibration is studied. In this section, we consider that the diversity phase used to create the 
B. Paul et al.: Coronagraphic phase diversity: performance study and laboratory demonstration

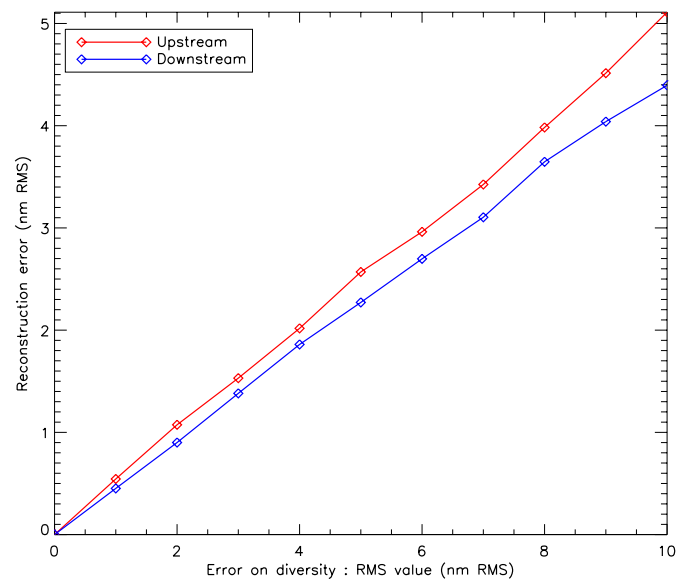

Fig. 4. Error reconstructions upstream (solid red line) and downstream (solid blue line) of the coronagraph as functions of the error on the diversity phase.

diversity image is not perfectly known. The coronagraphic simulated diversity image is computed with a diversity phase $\phi_{\text {div }}^{\prime}=$ $\phi_{\text {div }}+\phi_{\text {err }}$, with $\phi_{\text {err }}$ a randomly generated phase of given rms value, and COFFEE's phase reconstruction is done considering that the diversity phase is equal to $\phi_{\text {div }}$. In Fig. 4 , we see that the reconstruction error increases linearly with the calibration error on the diversity phase, with a slope of 0.5 . Thus, the requirement on the calibration precision for the diversity phase is typically the precision wanted for the aberration measurement.

\subsection{Impact of aliasing}

The phase estimation is performed here on a truncated Zernike basis. In real images (recorded from a bench), some speckles will originate in high-order aberrations. These aberrations, which cannot be fitted by the truncated Zernike basis, will have an impact on the phase estimation, called aliasing error hereafter. Thus, it is necessary to study this aliasing error as a function of the number of Zernike modes used in the phase reconstruction. Here, we generate a phase on a large number of Zernike modes, and compute the corresponding images using the perfect coronagraph model. Aberrations both upstream and downstream of the coronagraph are then estimated by COFFEE using an increasing number of Zernike modes. Since one of the aims of this simulation is to determine the size of the truncated Zernike basis to be used with experimental data recorded on an in-house bench, the noise level in the simulated images corresponds to the one we have on this bench. The total incoming flux is $5 \times 10^{6}$ photons, and the detector noise is $\sigma_{\operatorname{det}}=1 \mathrm{e}^{-}$per pixel. Parameters used for this simulation are gathered in Table 2. This simulation has been done with and without a regularization metric, so that we can demonstrate the relevance of this metric on phase estimation.

Figure 5 presents the evolution of the reconstruction errors when the number of reconstructed Zernike modes increases. Here, every reconstruction error (Eq. (13)) is calculated on a basis of 350 Zernike modes; thus, the error originates both in high-order aberrations, which are not considered by COFFEE because of the Zernike basis finite size (modelling error), and in the impact of these high-order aberrations on the estimated ones (aliasing). The WFE corresponding to the aberrations that are not estimated by COFFEE (from $N$ to 350, where $N$ varies between 15 and 275 according to Table 2) is called "unmodelled WFE" hereafter.
Table 2. COFFEE: simulation parameters for studying the aliasing error.

\begin{tabular}{|c|c|}
\hline \multicolumn{2}{|c|}{ Simulation } \\
\hline Image size & $\begin{array}{l}93 \times 93 \frac{\lambda}{D}(128 \times 128 \text { pixels, } \\
\text { oversampling factor: } 1.38)\end{array}$ \\
\hline Light spectrum & monochromatic $(\lambda=635 \mathrm{~nm})$ \\
\hline $\begin{array}{l}\text { Aberration upstream of the } \\
\text { coronagraph }\left(\phi_{\mathrm{u}}\right)\end{array}$ & $\mathrm{WFE}=80 \mathrm{~nm} \mathrm{rms}$ \\
\hline $\begin{array}{l}\text { Aberration downstream of } \\
\text { the coronagraph }\left(\phi_{\mathrm{d}}\right)\end{array}$ & $\mathrm{WFE}=20 \mathrm{~nm} \mathrm{rms}$ \\
\hline $\begin{array}{l}\text { Zernike basis used for } \phi_{\mathrm{u}} \\
\text { and } \phi_{\mathrm{d}} \text { simulation }\end{array}$ & 350 Zernike polynomials \\
\hline Incoming flux & $5 \times 10^{6}$ photons \\
\hline Noise & $\begin{array}{l}\text { photon noise, detector noise } \\
\left(\sigma_{\mathrm{det}}=1 \mathrm{e}^{-}\right)\end{array}$ \\
\hline \multicolumn{2}{|c|}{ COFFEE: phase estimation } \\
\hline $\begin{array}{l}\text { Zernike basis used for } \phi_{\mathrm{u}} \\
\text { and } \phi_{\mathrm{d}} \text { reconstruction }\end{array}$ & $\begin{array}{l}\text { from } 15 \text { to } 275 \text { Zernike } \\
\text { polynomials }\end{array}$ \\
\hline Regularization metric & With and without \\
\hline
\end{tabular}
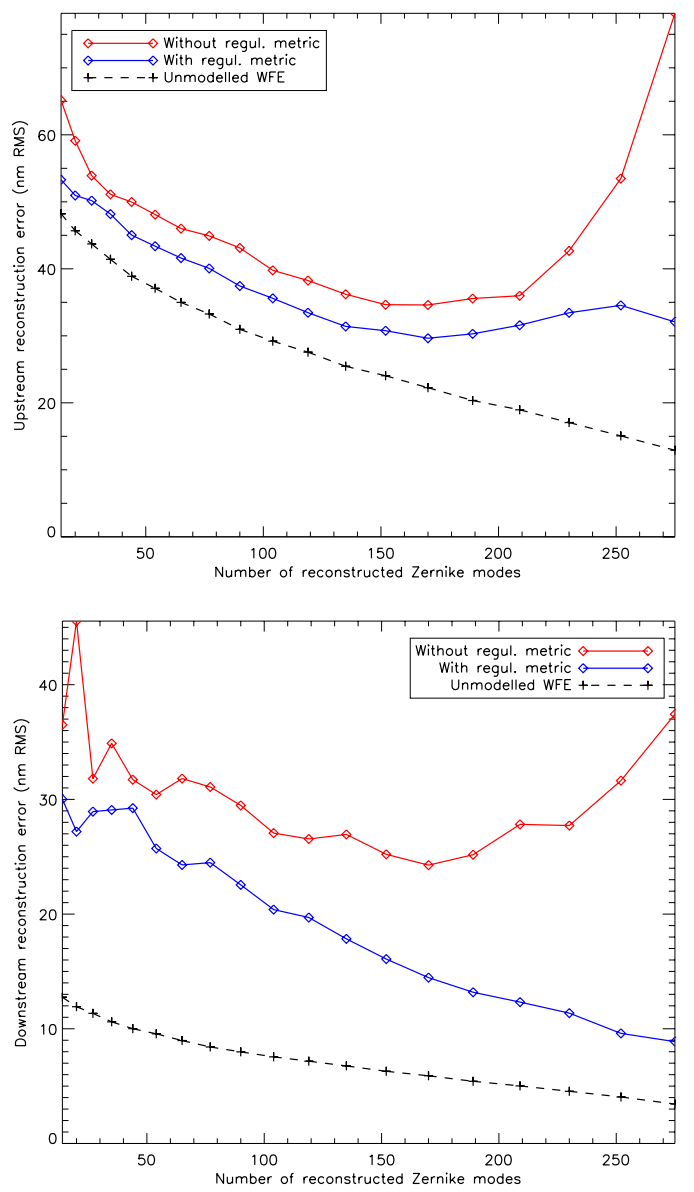

Fig. 5. Error reconstructions upstream (top) and downstream (bottom) of the coronagraph as functions of the number of reconstructed Zernike modes, with a regularization metric (solid blue line) and without (solid red line).

In the plot of the reconstruction error upstream of the coronagraph evolution (Fig. 5, top), one can see that without a regularization metric, the reconstruction error increases for a large number of Zernike modes. An interpretation of this behaviour is the following: because high-order aberrations have a smaller 


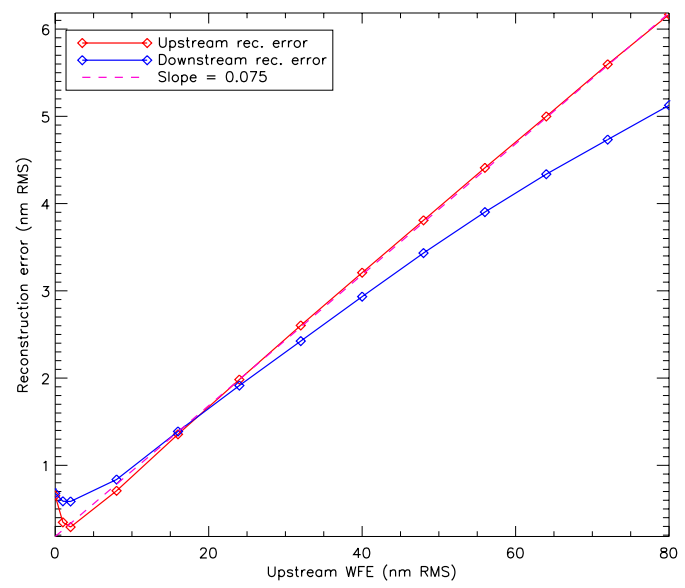

Fig. 6. Error reconstruction upstream of the coronagraph with respect to the WFE of the aberration upstream of the coronagraph.

variance, their associated speckle intensity is lower. Thus, owing to the photon and detector noise in the image, the SNR is smaller for these aberrations. Such behaviour leads to a trade-off between aliasing and noise amplification for the optimal number of Zernike modes (Fig. 5). The best number of Zernike modes is then a function of the aberrations level (WFE) and spectrum, as well as of the level of noise. The use of a regularization metric allows us to avoid this noise amplification (Fig. 5): the reconstruction error roughly reaches a saturation level (rather than growing to very high values). Additionally, the use of regularization reduces the aliasing error, and avoids the need for the difficult and somewhat ad hoc choice of number of Zernike modes for the reconstruction.

According to the results presented in Fig. 5, we have chosen to estimate the aberrations upstream and downstream of the coronagraph on 170 Zernike modes with the regularization metric of Eq. (4).

\subsection{Model mismatch}

We have already demonstrated that ARPM images are compatible with the perfect coronagraph model and therefore with COFFEE estimation in Sauvage et al. (2012). The Roddier \& Roddier phase mask (RRPM; Roddier \& Roddier 1997; Guyon et al. 1999) consists in a $\pi$ phase shifting mask slightly smaller than the Airy disk. Additionally, the use of a circular prolate function as entrance pupil apodization $\boldsymbol{\Phi}_{\mathrm{P}}$ (ARPM), proposed by Soummer et al. (2003), leads in a perfect case (no aberrations upstream of the coronagraph) to a total suppression of signal in the detector plane. In the simulations presented hereafter, realistic ARPM coronagraphic images are computed following Soummer et al. (2007) to consider an accurate numerical representation of Lyot-style coronagraphs. Then, we use COFFEE to reconstruct both phases upstream and downstream of the coronagraph. Here, when using the formalism developed in Sect. 2.2, the prolate apodization function $\boldsymbol{\Phi}_{\mathrm{P}}$ is included in both simulation and reconstruction imaging models.

Because the perfect coronagraph model is not exactly identical to an ARPM (although their responses to aberrations is very close), there is a model mismatch in the estimation of aberrations upstream of the coronagraph $\phi_{u}$, which varies linearly with the WFE of $\phi_{\mathrm{u}}$, as shown in Fig. 6. The model mismatch can thus be quantified as $7.5 \%$ of the WFE rms value of $\phi_{\mathrm{u}}$, except for very small WFE $(\leq 1 \mathrm{~nm} \mathrm{rms})$, where the variation is non-linear, but remains below $1 \mathrm{~nm}$ rms.

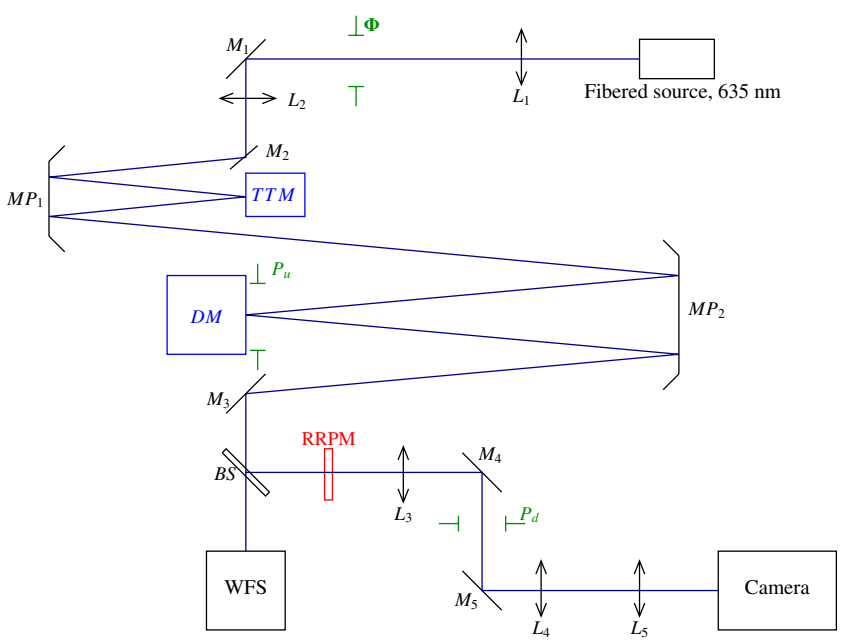

Fig. 7. Adaptive optics testbed schematic representation. $\mathrm{M}_{i}$ : fold mirrors; $\mathrm{MP}_{i}$ : parabolic mirrors; $\mathrm{L}_{i}$ : lenses (doublets); $\mathrm{BS}$ : beam splitter; TTM: Tip-Tilt mirror; DM: deformable mirror; RRPM: coronagraphic focal plane mask; $\boldsymbol{\Phi}$ : prolate apodizer; WFS: AO wave-front sensor

Since the variation in this model mismatch varies linearly with the WFE of $\boldsymbol{\phi}_{\mathrm{u}}$, it should not limit the ability to compensate for the aberration upstream of an ARPM using COFFEE as focal plane wave-front sensor (WFS).

\section{Laboratory demonstration}

In this section we present experimental validations in the coronagraphic phase diversity. These validations are done on the bench BOA, described in Sect. 4.1. Section 4.2 describes a carefully designed method developed to introduce calibrated static aberrations on the AO bench to be measured with COFFEE. The error made on the measurements of aberrations upstream of the coronagraph (NCPA) is quantified in Sect. 4.3. Section 4.4 presents the static aberration measurement performance, and Sect. 4.5 details the procedure for compensating for the measured aberrations.

\subsection{Experimental setup}

Figure 7 shows the design of our in-house bench. The input beam, emitted from a fibered laser source $(\lambda=635 \mathrm{~nm})$ comes through the prolate apodizer $\boldsymbol{\Phi}$, which is in the entrance pupil plane $\left(P_{\mathrm{u}}\right)$. The beam is reflected by the tip-tilt mirror (TTM) and then by the deformable mirror (DM, entrance pupil, $D_{\mathrm{u}}=$ $40 \mathrm{~mm}, 6 \times 6$ actuators). The beam-splitter sends a fraction of the beam to the AO wave-front sensor (Shack-Hartmann, $5 \times 5$ sub-apertures). On the other channel, the light is focused onto a RRPM, whose diameter is $d_{\mathrm{c}}=18.1 \mu \mathrm{m}$ (angular diameter is $\left.1.06 \frac{\lambda}{D_{\mathrm{u}}}\right)$. After going through the Lyot stop plane $\left(P_{\mathrm{d}}\right.$, with $\left.D_{\mathrm{d}}=0.99 D_{\mathrm{u}}\right)$, the beam is focused onto the camera $(256 \times 256$ pixels images with an oversampling of 2.75 , detector noise $\sigma_{\text {det }}=1 \mathrm{e}^{-}$). For faster computations, recorded images are re-binned to $128 \times 128$ pixels images with an oversampling of 1.38 .

\subsection{Introduction of calibrated aberrations}

To evaluate COFFEE's performance, we introduce calibrated aberrations on the bench using a process described in this section. We consider an aberration phase $\phi_{\text {cal }}$ to be introduced on 

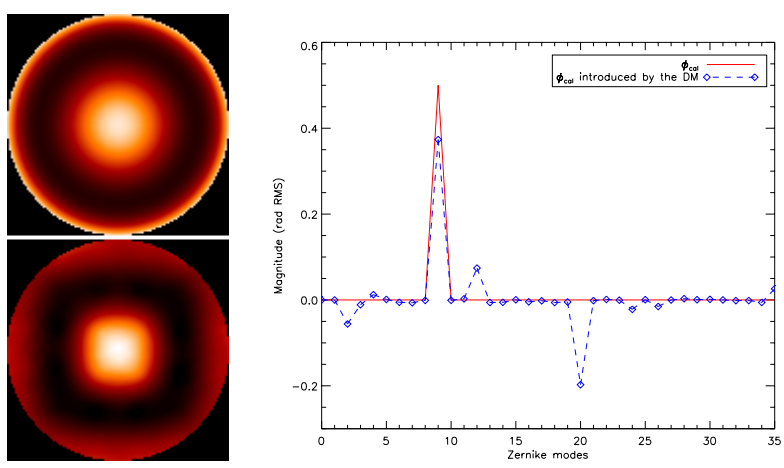

Fig. 8. Introduction of calibrated aberration on BOA: case of a pure spherical aberration. Left: theoretical wave-front (top) and DM introduced wave-front (bottom). Right: corresponding Zernike modes for the theoretical introduced aberration (solid red line) and the DM introduced aberration (dashed blue line).

BOA. First, since the phase is represented by the DM with a finite number of actuators $(6 \times 6)$, the introduced aberration will not match the aberration $\phi_{\text {cal }}$ perfectly, as illustrated in Fig. 8 in the case of a pure spherical aberration.

Our aim is here to introduce, using the DM, the closest aberration to the aberration $\phi_{\text {cal }}$. We let $\boldsymbol{F}$ be the DM influence matrix (obtained by calibration); any DM introduced aberration $\phi^{\mathrm{DM}}$ can be described as a set of actuator voltages $\boldsymbol{u}\left(\boldsymbol{\phi}^{\mathrm{DM}}=\boldsymbol{F} \boldsymbol{u}\right)$. We are thus looking for the set $\boldsymbol{u}_{\mathrm{cal}}$ which solves the least-squares problem:

$\boldsymbol{u}_{\mathrm{cal}}=\underset{\boldsymbol{u}}{\arg \min }\left\|\boldsymbol{F} \boldsymbol{u}-\boldsymbol{\phi}_{\mathrm{cal}}\right\|^{2}$.

The solution of this problem can be written as

$\boldsymbol{u}_{\mathrm{cal}}=\boldsymbol{T} \boldsymbol{\phi}_{\mathrm{cal}}$

with $\boldsymbol{T}$ the generalized inverse of matrix $\boldsymbol{F}$. Using the interaction matrix $\boldsymbol{D}$ (resulting from calibration), we can compute the corresponding set of slopes $\boldsymbol{s}_{\text {cal }}\left(\boldsymbol{s}_{\mathrm{cal}}=\boldsymbol{D} \boldsymbol{u}_{\mathrm{cal}}\right)$, which can then be used to modify the AO loop reference slopes $\boldsymbol{s}_{\text {ref }}$. Thus, closing the AO loop with the reference slopes $s_{\text {ref }}+s_{\text {cal }}$, we introduce an aberration $\boldsymbol{\phi}_{\text {cal }}^{\mathrm{DM}}=\boldsymbol{F} \boldsymbol{u}_{\mathrm{cal}}=\boldsymbol{F} \boldsymbol{T} \boldsymbol{\phi}_{\text {cal }}$ on the bench, which is the best fit of $\phi_{\text {cal }}$ in the least squares sense.

We also have to consider that the bench BOA presents its own unknown static aberrations $\phi_{\mathrm{u}}^{\mathrm{BOA}}$ and $\boldsymbol{\phi}_{\mathrm{d}}^{\mathrm{BOA}}$ upstream and downstream of the coronagraph (respectively). Thus, if a calibrated aberration $\phi_{\text {cal }}$ is introduced in the entrance pupil, aberrations $\phi_{\mathrm{u}}$ upstream of the coronagraph will be

$\phi_{\mathrm{u}}=\phi_{\mathrm{cal}}+\phi_{\mathrm{u}}^{\mathrm{BOA}}$.

To get rid of the unknown aberration $\phi_{\mathrm{u}}^{\mathrm{BOA}}$, we perform a differential phase estimation:

1. We introduce the aberration $\phi_{\mathrm{cal}}^{\mathrm{DM}}$ on the bench. A phase $\hat{\boldsymbol{\phi}}_{\mathrm{u}}^{+}=$ $\hat{\boldsymbol{\phi}}_{\mathrm{cal}}^{\mathrm{DM}}+\hat{\boldsymbol{\phi}}_{\mathrm{u}}^{\mathrm{BOA}}$ is estimated using focused and diverse images recorded on the camera.

2. The opposite aberration $-\boldsymbol{\phi}_{\mathrm{cal}}^{\mathrm{DM}}$ is then introduced. A phase $\hat{\boldsymbol{\phi}}_{\mathrm{u}}^{-}=-\hat{\boldsymbol{\phi}}_{\mathrm{cal}}^{\mathrm{DM}}+\hat{\boldsymbol{\phi}}_{\mathrm{u}}^{\mathrm{BOA}}$ is estimated.

3. The half difference $\hat{\boldsymbol{\phi}}_{\mathrm{cal}}^{\mathrm{DM}}=\frac{\hat{\boldsymbol{\phi}}_{\mathrm{u}}^{+}-\hat{\boldsymbol{\phi}}_{u}^{-}}{2}$ is our estimate of $\boldsymbol{\phi}_{\mathrm{cal}}$.

The first use of this process is to calibrate the diversity phase itself. Since this phase will be introduced using the AO system, the actually introduced diversity phase will not exactly match the theoretical mix of defocus and astigmatism. We introduce the aberrations $\phi_{\text {div }}$ and $-\phi_{\text {div }}$ on the bench using the AO system. These two aberrations are then estimated using classical phase diversity (no coronagraph), with a pure defocus of diversity phase introduced using a flat glass plate of known thickness $e$ in a focused beam.

Such a process gives us an accurate estimation of the diversity phase really introduced on the bench, with an estimated accuracy of $4 \mathrm{~nm}$ rms on the introduced aberration. This calibration is then used in COFFEE's estimations performed on experimental images.

\subsection{Performance assessment: error budget}

From simulations presented in Sect. 3, we establish an error budget for estimating aberrations upstream of the coronagraph using experimental data:

$\diamond$ Photon and detector noise error: on the BOA bench, the typical incoming flux is $f_{\mathrm{BOA}}=5 \times 10^{6}$ photons. Knowing that we have photon noise and a detector noise with $\sigma_{\text {det }}=1 \mathrm{e}^{-}$, we can evaluate the noise error: $\epsilon_{\text {noise }}=0.9 \mathrm{~nm}$ rms.

$\diamond$ The diversity phase $\phi_{\text {div }}$ has been calibrated using classical phase diversity, using the process presented in Sect. 4.2. Such an estimation has been performed with an error of $4.0 \mathrm{~nm}$ rms (value calculated from an error budget evaluated for a classical phase diversity estimation on the BOA bench. Such accuracy has already been obtained on this bench by Sauvage et al. 2007). According to Sect. 3.3, this error on the diversity phase leads to an error $\epsilon_{\text {model }}=$ $2.0 \mathrm{~nm} \mathrm{rms}$.

$\diamond$ The source is a coherent Gaussian-shaped beam whose FWHM is $0.27 \frac{\lambda}{D}$ on the coronagraph. According to the simulations of Sect. 3.2, this leads to a reconstruction error: $\epsilon_{\mathrm{obj}}=0.7 \mathrm{~nm} \mathrm{rms}$.

$\diamond$ Residual turbulent speckles, which originate in uncorrected turbulent aberrations, are not included in the imaging model. To measure the impact of these speckle on the reconstruction, several wave-fronts have been successively recorded using a commercial Shack-Hartmann wave-front sensor. From these acquisitions, we calculate the WFE of the residual turbulent phase: $\sigma_{\phi_{\text {turb }}}=1.2 \mathrm{~nm}$ rms. This residual turbulence will create speckles on the detector, which will be considered by COFFEE as originating in NCPA. Thus, the residual turbulence error $\epsilon_{\text {turb }}$ made by COFFEE is estimated to $\epsilon_{\text {turb }}=\sigma_{\phi_{\text {turb }}}=1.2 \mathrm{~nm} \mathrm{rms}$.

$\diamond$ Aliasing error, which originates in high-order aberrations, has been studied in Sect. 3.4. For a phase upstream of the coronagraph estimated on $N=170$ Zernike modes, we have $\epsilon_{\text {aliasing }}=18.3 \mathrm{~nm} \mathrm{rms}$.

$\diamond$ From simulations, we know that the model mismatch is $7.5 \%$ of WFE. For this study, we will not estimate aberrations with a WFE stronger than $80 \mathrm{~nm}$ rms. For such a WFE, the model error is $\epsilon_{\text {model }}=6.0 \mathrm{~nm} \mathrm{rms}$.

As one can see in Table 3, the error budget is mainly driven by the aliasing error. The second most important term is the model mismatch (even though it goes to zero with the WFE).

\subsection{Measurement of aberrations upstream of the coronagraph}

In this section, we introduce calibrated aberrations on the BOA bench upstream of the coronagraph, and then estimate them with 
Table 3. COFFEE: error budget for the estimation of an aberration upstream of the coronagraph on BOA.

\begin{tabular}{lc}
\hline \hline & Error budget \\
\hline Noise & $\epsilon_{\text {noise }}=0.9 \mathrm{~nm} \mathrm{rms}$ \\
Model mismatch & $\epsilon_{\text {model }}=6.0 \mathrm{~nm} \mathrm{rms}$ \\
Error on diversity & $\epsilon_{\text {div }}=2.0 \mathrm{~nm} \mathrm{rms}$ \\
Resolved object & $\epsilon_{\text {obj }}=0.7 \mathrm{~nm} \mathrm{rms}$ \\
Residual turbulence & $\epsilon_{\text {turb }}=1.2 \mathrm{~nm} \mathrm{rms}$ \\
Aliasing & $\epsilon_{\text {aliasing }}=18.3 \mathrm{~nm} \mathrm{rms}$ \\
\hline Total error & $\epsilon=\sqrt{\sum_{i} \epsilon_{i}^{2}}=20.6 \mathrm{~nm} \mathrm{rms}$ \\
Total error per Zernike & $\epsilon^{\prime}=1.6 \mathrm{~nm} \mathrm{rms} \mathrm{per} \mathrm{estimated}$ \\
mode & Zernike mode \\
\hline
\end{tabular}

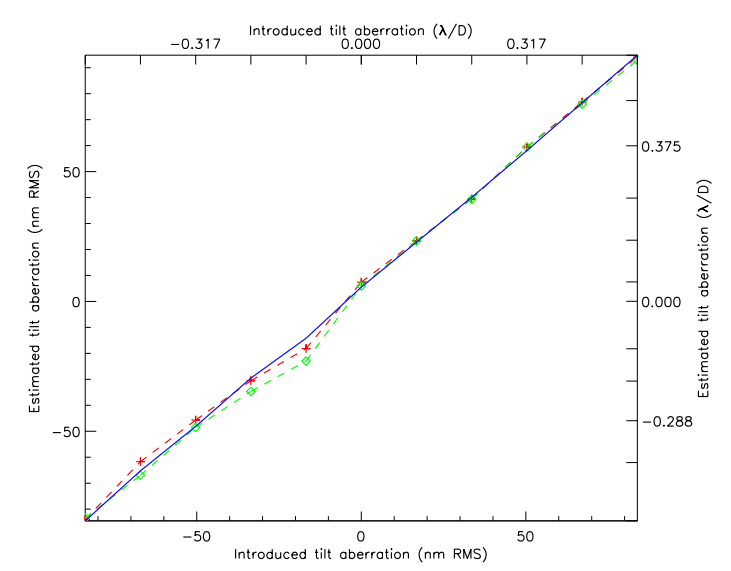

Fig. 9. Estimation of a tilt aberration on BOA: calibration (solid blue line) and COFFEE's estimation with bound on the tip-tilt downstream of the coronagraph (dashed crossed red line) and without boundaries (dashed diamond green line).

COFFEE in order to evaluate its performance. In the course of this study, we realized that the position of the coronagraphic image on the detector (quantified by the tip-tilt downstream of the coronagraph) is a critical issue. Indeed, it occurred that COFFEE was able to perform phase retrieval only for downstream tiptilt $\left[a_{2}, a_{3}\right]$ values within the range $[-100 \mathrm{~nm} \mathrm{rms} ; 100 \mathrm{~nm} \mathrm{rms}]$ $\left(\left[-\frac{\lambda}{6 D} ; \frac{\lambda}{6 D}\right]\right)$. To get rid of this constraint, we have developed a method to perform a preliminary estimation of the tip-tilt downstream of the coronagraph. This method, which uses the diversity image, is fully described in Appendix B.

\subsubsection{Measurement of tip-tilt upstream of the coronagraph}

We present the estimation of a tilt aberration upstream of the coronagraph using COFFEE in this section. Using the AO system, we introduce a tilt aberration by adding a constant value $\delta \boldsymbol{s}_{\mathrm{TT}}$ to the AO wave-front sensor references slopes $s_{\text {ref }}$, and then closing the AO loop on the slopes $\boldsymbol{s}_{\text {ref }}+\delta \boldsymbol{s}_{\mathrm{TT}}$. To accurately calibrate the introduced tilt, for each position, we first estimate the aberrations using classical phase diversity (no coronagraph). Then, the RRPM is put in the focal plane, and the same operation is repeated: for each position, we record two images, and then estimate the aberrations using COFFEE.

From the upstream tilt reconstruction performed by COFFEE (Fig. 9), we calculate an average reconstruction error: $\epsilon_{\text {tilt }}=2.1 \mathrm{~nm}$. Part of this error is due to an error on the estimation of tip-tilt downstream of the coronagraph. An improved estimation has been performed by setting boundaries on the downstream tip-tilt. Its value is evaluated before COFFEE's estimation using the method described in Appendix B with the diversity coronagraphic image recorded for a tip-tilt upstream the coronagraph value close to $0 \mathrm{~nm}$ rms (centered coronagraph). Such an estimation process gives us an estimation of tip-tilt downstream of the coronagraph $\left\{a_{2}^{\text {do }}, a_{3}^{\text {do }}\right\}$ with an accuracy of $\pm 1.5 \mathrm{~nm}$ rms. Using this estimation as the starting value for the minimization, and setting bounds of $\pm 1.5 \mathrm{~nm} \mathrm{rms}$ on it, we processed the same experimental data. This, in turn, results in a better estimation of tilt upstream of the coronagraph (Fig. 9), with an average error $\epsilon_{\text {tilt }}=1.5 \mathrm{~nm}$, which is close to the expected error per Zernike mode given in Sect. 4.3 $\left(\epsilon^{\prime}=1.6 \mathrm{~nm} \mathrm{rms}\right)$.

\subsubsection{NCPA measurements}

In this section, we introduce aberrations upstream of the coronagraph. The aberration $\phi_{\text {cal }}$ is expanded on the first 15 Zernike modes (which is the largest number of modes we can properly describe with our $6 \times 6 D M)$, and then we estimate these aberrations using COFFEE, following the process described in Sect. 4.2. To take the DM action into account on the introduced phase (illustrated in Fig. 8), aberrations $\phi_{\text {cal }}$ are first estimated with classical phase diversity (no phase mask in the coronagraphic focal plane, Sauvage et al. 2007). This estimation gives us a calibration of the introduced aberration, which is then used to evaluate the accuracy of COFFEE's estimation.

At convergence of the reconstruction, a very good match can be observed between the experimental images and the ones computed for the estimated aberrations (Fig. 10, top and middle). This, in turn, results in a very good match between the aberrations measured by COFFEE (Fig. 10, right) and the introduced ones (Fig. 10, left).

From the experimental phase estimation presented in Fig. 10, we compute a reconstruction error between the classical diversity phase calibrated aberration and COFFEE's estimation:

$\epsilon_{\text {exp }}=22.5 \mathrm{~nm} \mathrm{rms}$.

One can notice that this error is close to the expected error budget, i.e. that there is a good match between the performance assessment study carried out in Sect. 3 and the experimental results presented in this section.

\subsection{Low-order NCPA compensation}

Lastly, the ability of COFFEE to compensate for the aberrations upstream of the coronagraph is experimented on BOA. In Sect. 4.4, the aberrations upstream of the coronagraph are expanded on 170 Zernike modes, in order to have the smallest reconstruction error (according to Sect. 3.4).

As previously mentioned, the compensation on BOA is limited to the 15 th Zernike mode. Thus, what is required in a closed loop process is the most accurate estimation of 15 Zernike modes rather than an accurate measurement of every estimated Zernike mode. Using a basis of 36 Zernike modes for the reconstruction is sufficient to give an accurate estimation of the first 15 Zernike modes: the aliasing error, which is the most important error source, will mainly degrade the estimation accuracy of the reconstructed high orders (close to $Z_{36}$ ).

To demonstrate the ability of COFFEE to be used in a closed loop, we introduce a set of aberrations on the $D M$ by modifying the reference slopes, as described in Sect. 4.2. Then, we 
B. Paul et al.: Coronagraphic phase diversity: performance study and laboratory demonstration
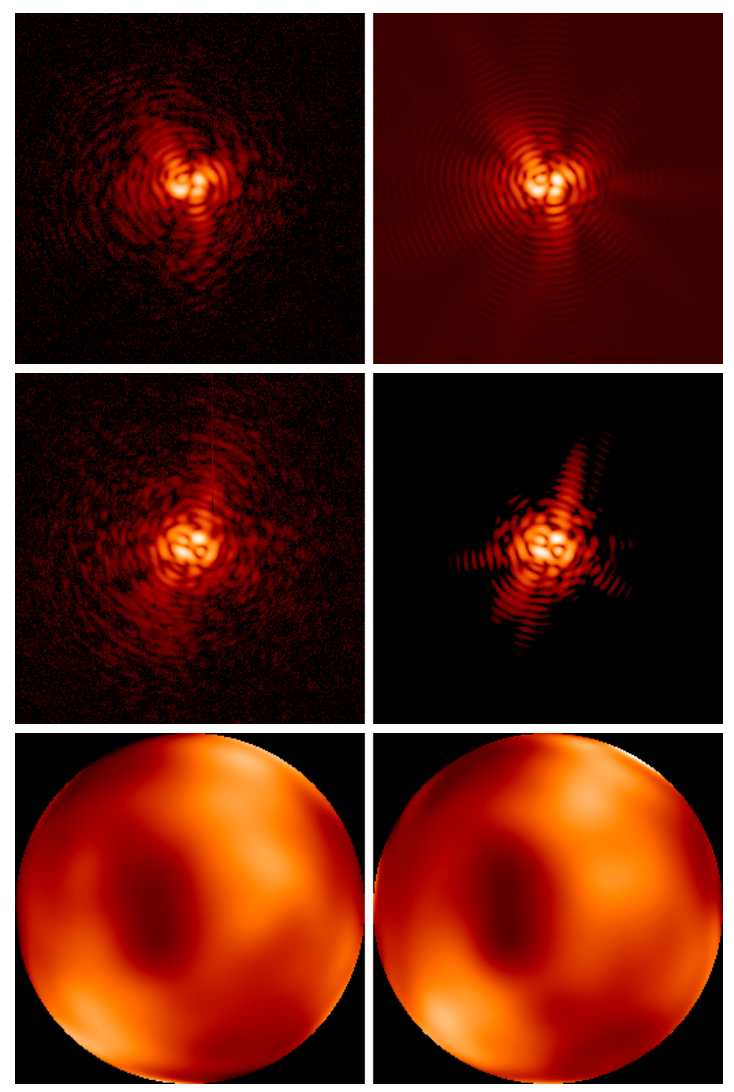

Fig. 10. COFFEE: NCPA estimation of an introduced phase $\phi_{\text {cal }}$ on BOA. Top: for an aberration $+\phi_{\text {cal }}$, recorded coronagraphic image from the bench (left) and computed image using the reconstructed aberration $\hat{\boldsymbol{\phi}}_{\mathrm{u}}^{+}$(right) (log. scale, same range for both images). Middle: same images for an aberration $-\boldsymbol{\phi}_{\text {cal }}$ introduced and a reconstructed aberration $\hat{\phi}_{\mathrm{u}}^{-}$(log. scale, same range for both images). Bottom: calibrated introduced aberration (left) and COFFEE estimated aberration (right).

use the pseudo-closed loop (PCL) method described in Sauvage et al. (2007). This iterative process has two stages: for the PCL iteration $i$ :

1. acquisition of the focused $\boldsymbol{i}_{\mathrm{c}}^{f}$ and diverse $\boldsymbol{i}_{\mathrm{d}}^{f}$ images;

2. estimation of the aberration $\hat{\boldsymbol{\phi}}_{\mathrm{u}}^{i}$ upstream of the coronagraph;

3 . computation of the corresponding reference slopes correction $\delta \boldsymbol{s}=g \boldsymbol{D} \boldsymbol{T} \hat{\boldsymbol{\phi}}_{\mathrm{u}}^{i}$, where $\boldsymbol{D}$ and $\boldsymbol{T}$ are the interaction and influence matrices defined in Sect. 4.2 and $g$ is the PCL gain;

4. the AO loop is closed on the modified reference slopes.

The computation time ( $\operatorname{step} 2$ ) varies from $1 \mathrm{~min}$ to $2.5 \mathrm{~min}$, allowing us to compensate for quasi-static aberrations upstream of the coronagraph. This compensation process is limited by the estimation accuracy of the first 15 Zernike modes performed by COFFEE, which corresponds to the error budget established in Sect. 4.3), and by the ability of the DM to reproduce a given wave-front. Indeed, the correction introduced on the bench (step 2 of the PCL process) is the best fit of the estimated phase $\hat{\boldsymbol{\phi}}_{\mathrm{u}}^{i}$ in the least-square sense (as presented in Sect. 4.2). The difference between the estimated aberration and the actual introduced correction will thus limit the compensation performance of the PCL process. Considering these two limitations, one can compute the variance $\sigma_{\mathrm{BOA}}^{2}$ (for the first 15 Zernike modes) that can be reached on the BOA bench:

$\sigma_{\mathrm{BOA}}^{2}=4.4 \times 10^{-2}{\mathrm{rad} \mathrm{rms}^{2} .}^{2}$

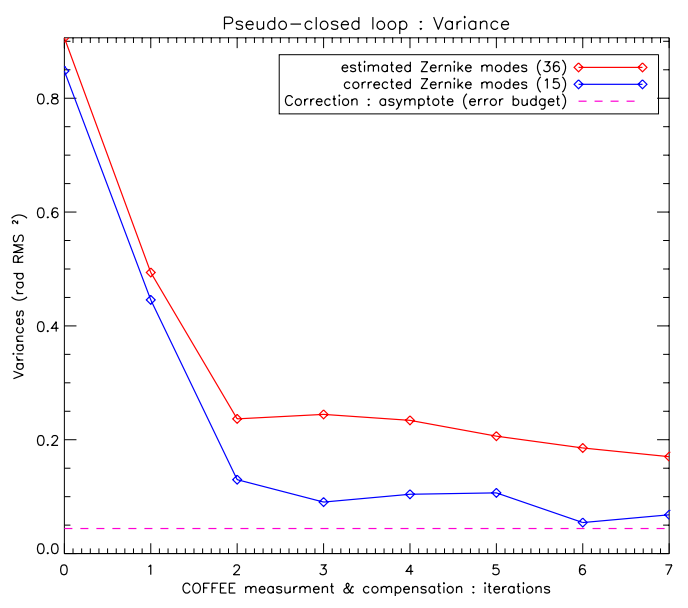

Fig. 11. PCL on the bench BOA $\left(g_{\mathrm{PCL}}=0.5\right)$ : variance of the residual static aberrations upstream of the coronagraph for the 36 COFFEE estimated Zernike modes (solid red line) and the 15 corrected modes (solid blue line). The magenta dashed line represents the ultimate performance one can reach according to the error budget detailed in 4.3.

The correction and stabilization of the NCPA variance can be seen in Fig. 11. One can see that the variance of the 15 corrected Zernike modes reaches the expected asymptotic value $\sigma_{\text {BOA }}^{2}$. This result is the very first demonstration of COFFEE's ability to compensate for aberrations upstream of the coronagraph. A compensation at levels compatible with SPHERE or GPI-like instruments will require using a DM with many more actuators, and working on the reduction of the dominant term of the error budget, which is aliasing.

\section{Conclusion}

In this paper, we have presented a thorough simulation study (Sect. 3) and a first experimental validation (Sect. 4) of the coronagraphic wave-front sensor called COFFEE, which consists mainly in the extension of the phase diversity concept to a coronagraphic imaging system. From the validation and careful performance assessment of COFFEE, we showed that COFFEE is currently limited by the aliasing error, due to high-order aberrations, which are difficult to model with a Zernike basis.

In Sect. 4, we presented a first experimental validation of COFFEE using an ARPM. We introduced calibrated aberrations upstream of the coronagraph (NCPA), using the AO sub-system, and estimated them with COFFEE. The accuracy we obtained on these estimation shows a very good match with our error budget. Lastly, we used COFFEE in an iterative process to perform a preliminary validation of COFFEE's ability to compensate for the aberrations upstream of the coronagraph.

Several perspectives are currently considered to optimize COFFEE: firstly, in order to minimize the impact of the aliasing error on the phase reconstruction, we plan to perform the phase reconstruction on a pixel-wise map, which is more suitable than a truncated Zernike basis. Secondly, we would like to improve the imaging model, both to make COFFEE work with other coronagraph than the ARPM and to reduce the model error, which is currently the second most important one, even though it goes to zero with the WFE. Two solutions are considered. In the absence of residual turbulence, an accurate imaging model is obtained by propagating the electric field through each plane of the coronagraphic imaging system (Fig. 1) for an arbitrary focal plane coronagraphic mask. Such a method, where no model error needs to be considered, can be used for a laboratory calibration. 
Alternatively, a more accurate analytical imaging model, which could include a residual turbulent aberration, can be developed. Such a model, which could include a residual turbulent aberration, will ultimately allow us to perform NCPA estimation on images from the sky. These improvements should allow us to estimate and compensate for the aberrations upstream of the coronagraph using COFFEE with a nanometric precision in a closed loop process.

A further perspective is to extend COFFEE to phase and amplitude aberration estimation, in order to create a dark hole region in the coronagraphic image.

Acknowledgements. The authors would like to thank Mamadou N'Diaye, Kjetil Dohlen and Thierry Fusco for stimulating discussions, as well as Marc Ferrari, David Mouillet and Jean-Luc Beuzit for their support, and the Région ProvenceAlpes-Côte d'Azur for partial financial support of B.P. scholarship. This work has been partially funded by the European Commission under FP7 Grant Agreement No. 312430 Optical Infrared Coordination Network for Astronomy.

\section{Appendix A: Implementation details}

COFFEE performs a phase estimation by minimizing a criterion $J$ whose expression is given by Eq. (3). To estimate $\phi_{\mathrm{u}}$ and $\phi_{\mathrm{d}}$ (expanded on a truncated Zernike basis), we need both gradients $\frac{\partial J}{\partial a_{\mathrm{u}}}$ and $\frac{\partial J}{\partial \boldsymbol{a}_{\mathrm{d}}}$, where $\boldsymbol{a}_{x}=\left\{a_{x_{1}}, a_{x_{2}}, \ldots, a_{x_{N}}\right\}$ is a vector that contains the Zernike coefficients, for an aberration expanded on $N$ Zernike modes ( $x$ is for u (upstream) or d (downstream)).

Let us write the numerical expression of $J^{\text {foc }}$, using the notations defined in Sect. 2.1:

$$
\begin{aligned}
J= & \frac{1}{2} \sum_{t}\left|\frac{\boldsymbol{i}_{\mathrm{c}}^{\mathrm{foc}}[t]-\alpha \cdot \boldsymbol{h}_{\mathrm{det}}[t] \star \boldsymbol{h}_{\mathrm{c}}^{\mathrm{foc}}[t]-\beta}{\boldsymbol{\sigma}_{\mathrm{n}}^{\mathrm{foc}}[t]}\right|^{2} \\
& +\frac{1}{2} \sum_{t}\left|\frac{\boldsymbol{i}_{\mathrm{c}}^{\mathrm{div}}[t]-\alpha \cdot \boldsymbol{h}_{\mathrm{det}}[t] \star \boldsymbol{h}_{\mathrm{c}}^{\mathrm{div}}[t]-\beta}{\sigma_{\mathrm{n}}^{\mathrm{div}}[t]}\right|^{2} \\
& +\mathcal{R}_{\phi_{\mathrm{u}}}+\mathcal{R}_{\phi_{\mathrm{d}}} \\
= & J^{\mathrm{foc}}+J^{\mathrm{div}}+\mathcal{R}_{\phi_{\mathrm{u}}}+\mathcal{R}_{\phi_{\mathrm{d}}} .
\end{aligned}
$$

With $t$ the pixel position in the detector plane. $\sigma_{\mathrm{n}}^{\text {foc }}$ and $\sigma_{\mathrm{n}}^{\text {div }}$ are the noise variance maps. Considering the expression of $J$, we derive $J^{\text {foc }}$, and then deduce the gradients expressions of $J^{\mathrm{div}}$ using a trivial substitution. Expressions of the regularization terms gradients $\frac{\partial \mathcal{R}_{\phi_{\mathrm{x}}}}{\partial a_{\mathrm{x}}}$ are given by

$$
\frac{\partial \mathcal{R}_{\phi_{\mathrm{x}}}}{\partial \boldsymbol{a}_{\mathrm{x}}}=R_{\boldsymbol{a}_{\mathrm{x}}}^{-1} \boldsymbol{a}_{\mathrm{x}}
$$

The calculation of gradients $\frac{\partial J}{\partial \phi_{\mathrm{u}}}$ and $\frac{\partial J}{\partial \phi_{\mathrm{d}}}$ is done following Mugnier et al. (2001): first, we calculate the gradient of $J^{\mathrm{f}}$ with respect to the PSF $\boldsymbol{h}_{\mathrm{c}}$ :

$\frac{\partial J^{\mathrm{foc}}}{\partial \boldsymbol{h}_{\mathrm{c}}^{\mathrm{foc}}}=\frac{1}{\boldsymbol{\sigma}_{\mathrm{n}}^{\mathrm{foc}^{2}}}\left[\alpha \boldsymbol{h}_{\mathrm{det}}\left(\alpha \cdot \boldsymbol{h}_{\mathrm{det}} \star \boldsymbol{h}_{\mathrm{c}}^{\mathrm{foc}}-\boldsymbol{i}_{\mathrm{c}}^{\mathrm{foc}}\right)\right]$.

Then, the calculation consists in derivating the gradient of the PSF $\boldsymbol{h}_{\mathrm{c}}$ with respect to phases $\boldsymbol{\phi}_{\mathrm{u}}[k]$ and $\boldsymbol{\phi}_{\mathrm{d}}[l]$ at pixels $k, l$ in pupils upstream and downstream of the coronagraph, respectively, and applying the chain rule, as already done in a non-coronagraphic case, e.g. in Thiébaut \& Conan (1995). The calculation of both gradients $\frac{\partial J^{\mathrm{foc}}}{\partial \phi_{\mathrm{u}}[k]}$ and $\frac{\partial J^{\mathrm{foc}}}{\partial \phi_{\mathrm{d}}[l]}$ gives

$$
\begin{aligned}
\frac{\partial J^{\mathrm{foc}}}{\partial \boldsymbol{\phi}_{\mathrm{u}}[k]}= & 2 \mathfrak{J}\left\{\boldsymbol{\psi}^{*}[k]\left[\mathrm{FT}\left(\frac{\partial J^{\mathrm{foc}}}{\partial \boldsymbol{h}_{\mathrm{c}}^{\mathrm{foc}}}\left(\boldsymbol{\Psi}-\eta_{0} \boldsymbol{\Psi}_{\mathrm{d}}\right)\right)\right]\right\}[k] \\
& -2 \Re\left(\frac{\partial \eta_{0}}{\partial \boldsymbol{\phi}_{\mathrm{u}}[k]} \sum_{t} \frac{\partial J^{\mathrm{f}}}{\partial \boldsymbol{h}_{\mathrm{c}}^{\mathrm{foc}}} \boldsymbol{\Psi}^{*} \boldsymbol{\Psi}_{\mathrm{d}}\right) \\
& +\frac{\partial\left|\eta_{0}\right|^{2}}{\partial \boldsymbol{\phi}_{\mathrm{u}}[k]} \sum_{t} \frac{\partial J^{\mathrm{f}}}{\partial \boldsymbol{h}_{\mathrm{c}}^{\mathrm{foc}}}\left|\boldsymbol{\Psi}_{\mathrm{d}}\right|^{2} \\
\frac{\partial J^{\mathrm{foc}}}{\partial \boldsymbol{\phi}_{\mathrm{d}}[l]}= & 2 \mathfrak{J}\left(\left(\boldsymbol{\psi}^{*}[l]-\eta_{0}^{*} \boldsymbol{\psi}_{\mathrm{d}}^{*}[l]\right)\right. \\
& \left.\times\left\{\mathrm{FT}\left[\frac{\partial J^{\mathrm{f}}}{\partial \boldsymbol{h}_{\mathrm{c}}^{\mathrm{foc}}}\left(\boldsymbol{\Psi}-\eta_{0} \boldsymbol{\Psi}_{\mathrm{d}}\right)\right]\right\}[l]\right) .
\end{aligned}
$$

With $\mathfrak{I}$ and $\mathfrak{R}$ the imaginary and real part (respectively), and

$$
\begin{aligned}
& \frac{\partial \eta_{0}}{\partial \boldsymbol{\phi}_{\mathrm{u}}}=j \boldsymbol{P}_{\mathrm{u}}^{2} \mathrm{e}^{j \phi_{\mathrm{u}}} \\
& \psi\left(\boldsymbol{\phi}_{\mathrm{u}}, \boldsymbol{\phi}_{\mathrm{d}}\right)=\boldsymbol{P}_{\mathrm{d}} \mathrm{e}^{j\left(\phi_{\mathrm{u}}+\boldsymbol{\phi}_{\mathrm{d}}\right)} \quad \boldsymbol{\Psi}\left(\boldsymbol{\phi}_{\mathrm{u}}, \boldsymbol{\phi}_{\mathrm{d}}\right)=\mathrm{FT}^{-1}(\psi) \\
& \boldsymbol{\psi}_{\mathrm{d}}\left(\boldsymbol{\phi}_{\mathrm{d}}\right)=\boldsymbol{P}_{\mathrm{d}} \mathrm{e}^{j \phi_{\mathrm{d}}} \quad \boldsymbol{\Psi}_{\mathrm{d}}\left(\boldsymbol{\phi}_{\mathrm{d}}\right)=\mathrm{FT}^{-1}\left(\boldsymbol{\psi}_{\mathrm{d}}\right) .
\end{aligned}
$$

Since the phases are expanded on a Zernike basis, we need the gradients of $J^{\text {foc }}$ with respect to the Zernike coefficients $a_{\mathrm{x}_{i}}$ of phase $\phi_{\mathrm{x}}$. These gradients are given by the expression (Mugnier et al. 2001):

$\frac{\partial J^{\mathrm{foc}}}{\partial a_{\mathrm{x}_{i}}}=\sum_{k} \frac{\partial J^{\mathrm{foc}}}{\partial \phi_{\mathrm{x}}[k]} Z_{i}[k]$

Flux $\alpha$ and constant background $\beta$ are also analytically estimated during the minimization, considering that

$J^{\mathrm{p}}[t]=\frac{1}{2} \sum_{t}\left|\frac{-\boldsymbol{i}_{\mathrm{c}}^{\mathrm{p}}[t]+\alpha \cdot \boldsymbol{h}_{\mathrm{det}}[t] \star \boldsymbol{h}_{\mathrm{c}}^{\mathrm{p}}[t]+\beta}{\boldsymbol{\sigma}_{\mathrm{n}}^{\mathrm{p}}[t]}\right|^{2}$

where p is for "foc" (focused) or "div" (diverse). For the sake of simplicity, we shall omit the variable $t$. We have

$$
\begin{aligned}
\frac{\partial J^{\mathrm{p}}}{\partial \alpha}= & \alpha \sum \frac{\left(\boldsymbol{h}_{\mathrm{det}} \star \boldsymbol{h}_{\mathrm{c}}^{\mathrm{p}}\right)^{2}}{\boldsymbol{\sigma}_{\mathrm{n}}^{\mathrm{p}^{2}}}+\beta \sum \frac{\boldsymbol{h}_{\mathrm{det}} \star \boldsymbol{h}_{\mathrm{c}}^{\mathrm{p}}}{\boldsymbol{\sigma}_{\mathrm{n}}^{\mathrm{p}^{2}}} \\
& -\sum \frac{\left(\boldsymbol{h}_{\mathrm{det}} \star \boldsymbol{h}_{\mathrm{c}}^{\mathrm{p}}\right) \boldsymbol{i}_{\mathrm{c}}^{\mathrm{p}}}{\boldsymbol{\sigma}_{\mathrm{n}}^{\mathrm{p}^{2}}} \\
\frac{\partial J^{\mathrm{p}}}{\partial \beta}= & \alpha \sum \frac{\boldsymbol{h}_{\mathrm{det}} \star \boldsymbol{h}_{\mathrm{c}}^{\mathrm{p}}}{\boldsymbol{\sigma}_{\mathrm{n}}^{\mathrm{p}^{2}}}+\beta \sum \frac{1}{\sigma_{\mathrm{n}}^{\mathrm{p}^{2}}}-\sum \frac{\boldsymbol{i}_{\mathrm{c}}^{\mathrm{p}}}{\boldsymbol{\sigma}_{\mathrm{n}}^{\mathrm{p}^{2}}}
\end{aligned}
$$

Which gives us, in a matricial form:

$$
\left(\begin{array}{cc}
\sum \frac{\left(\boldsymbol{h}_{\mathrm{det}} \star \boldsymbol{h}_{\mathrm{c}}^{\mathrm{p}}\right)^{2}}{\boldsymbol{\sigma}_{\mathrm{n}}^{\mathrm{p} 2}} & \sum \frac{\boldsymbol{h}_{\mathrm{det}} \star \boldsymbol{h}_{\mathrm{c}}^{\mathrm{p}}}{\boldsymbol{\sigma}_{\mathrm{n}}^{\mathrm{p} 2}} \\
\sum \frac{\boldsymbol{h}_{\mathrm{det}} \star \boldsymbol{h}_{\mathrm{c}}^{\mathrm{p}}}{\boldsymbol{\sigma}_{\mathrm{n}}^{\mathrm{p}^{2}}} & \sum \frac{1}{\boldsymbol{\sigma}_{\mathrm{n}}^{\mathrm{p} 2}}
\end{array}\right)\left(\begin{array}{c}
\alpha \\
\beta
\end{array}\right)=\left(\begin{array}{c}
\sum \frac{\left(\boldsymbol{h}_{\mathrm{det}} \star \boldsymbol{h}_{\mathrm{c}}^{\mathrm{p}}\right) i_{\mathrm{c}}^{\mathrm{p}}}{\sigma_{i}^{\mathrm{p} 2}} \\
\sum \frac{i_{\mathrm{c}}^{\mathrm{p}}}{\boldsymbol{\sigma}_{\mathrm{n}}^{\mathrm{p} 2}}
\end{array}\right) .
$$

A simple matrix inversion gives us the analytical estimation of the flux $\alpha$ and the background $\beta$ for each iteration. 

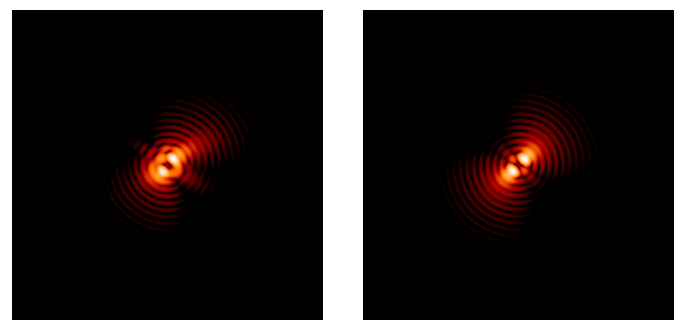

Fig. B.1. Coronagraphic diversity images computed for an aberration $\phi_{\mathrm{u}}+\phi_{\text {div }}$ upstream, $\phi_{\mathrm{d}}$ downstream of (left) and the only diversity aberration $\boldsymbol{\phi}_{\text {div }}(r i g h t)$. The shape of both images is mainly driven by diversity aberration.

\section{Appendix B: Tip-tilt estimation downstream of the coronagraph}

The tip-tilt downstream of the coronagraph (which represents the image position on the detector) strongly limits COFFEE's performance. Indeed, we determine that the phase estimation was accurate when $-100 \mathrm{~nm} \mathrm{rms} \leq a_{i} \leq 100 \mathrm{~nm} \mathrm{rms}$, with $a_{i}$ the Zernike coefficient for tip or tilt $(i \in\{2,3\})$. Beyond this range, COFFEE is unable to properly estimate both phases $\phi_{\mathrm{u}}$ and $\phi_{\mathrm{d}}$. Such a phenomenon strongly limits COFFEE's performance on a bench, since its utilization requires a restrictive location of the PSF on the detector.

To get rid of this limitation, we have developed a simple and fast method of estimating the tip-tilt downstream of the coronagraph before COFFEE's estimation, based on the diversity image. This image is created by adding a known aberration $\phi_{\text {div }}=a_{4}^{\text {div }} Z_{4}+a_{5}^{\text {div }} Z_{5}\left(a_{4}^{\text {div }}=a_{5}^{\text {div }}=80 \mathrm{~nm} \mathrm{rms}\right)$ to $\phi_{\mathrm{u}}$. Since the amplitude of this aberration is important $\left(\sigma_{\phi_{\text {div }}}=113 \mathrm{~nm}\right.$ $\mathrm{rms}$ ), the speckles we have in the coronagraphic diversity image mainly originate in this diversity aberration. This is illustrated in Fig. B.1, where we show two diversity images: one computed with randomly generated phases $\phi_{\mathrm{u}}$ (WFE $30 \mathrm{~nm} \mathrm{rms),} \phi_{\mathrm{d}}$ (WFE $10 \mathrm{~nm} \mathrm{rms}$ ), and another computed with no aberrations other than the diversity ones.

As one can see in Fig. B.1, we can clearly identify the aberrations which originate in the diversity $\phi_{\text {div }}$. The principle of our method lies in the research of these well-known aberrations (since we know the phase $\phi_{\text {div }}$ we introduce) in the diversity image $i_{\mathrm{c}}^{\mathrm{d}}$ by comparing it with a theoretical diversity image $i_{\mathrm{c}_{t h}}^{\mathrm{d}}$, calculated with no other aberrations than the diversity ones:

$\boldsymbol{i}_{\mathrm{c}_{t h}}^{\mathrm{d}}=\boldsymbol{h}_{\mathrm{det}} \star \boldsymbol{h}_{\mathrm{c}}\left(\boldsymbol{\phi}_{\mathrm{div}}, \boldsymbol{\phi}_{\mathrm{d}}=0\right)$.

The comparison of $i_{\mathrm{c}_{t h}}^{\mathrm{d}}$ with $i_{\mathrm{c}}^{\mathrm{d}}$ is performed using the method developed by Gratadour et al. (2005), which consists in minimizing the following criterion $J_{\mathrm{TT}}$

$J_{\mathrm{TT}}(x, y)=\left\|\frac{\boldsymbol{i}_{\mathrm{c}}^{\mathrm{div}}\left(x_{o}, y_{o}\right)-\boldsymbol{i}_{\mathrm{c}_{t h}}^{\mathrm{div}}\left(x_{o}, y_{o}\right) \star \delta\left(x_{o}-x, y_{o}-y\right)}{\sigma_{\mathrm{n}}^{\mathrm{div}}}\right\|^{2}$, where $\delta$ is the dirac function. Minimization of $J_{\text {TT }}$ gives us the shift $\left[x_{M}, y_{M}\right]$ between both images. It is then possible to calculate the corresponding tip $\left(a_{2}\right)$ and tilt $\left(a_{3}\right)$ downstream of the coronagraph knowing the image sampling $s$ :

$a_{2}=\frac{\pi}{2 s} x_{M} \quad a_{3}=\frac{\pi}{2 s} y_{M}$.

Finally, these estimated tip-tilt values are given to COFFEE as an input of the minimization, and are used as initial values to begin phase reconstruction. This method performs, on our experimental images, a fast preliminary estimation $(\sim 1 \mathrm{~s}$ for a $256 \times$ 256 image) of the tip-tilt downstream of the coronagraph with an accuracy of $1.5 \mathrm{~nm}$ rms, which is far enough, compared to the level of accuracy $( \pm 100 \mathrm{~nm} \mathrm{rms})$ required by COFFEE.

\section{References}

Baudoz, P., Boccaletti, A., Baudrand, J., \& Rouan, D. 2006, in Direct Imaging of Exoplanets: Science \& Techniques, Proc, IAU Colloq. 200, eds. C. Aime, \& F. Vakili (Cambridge, UK: Cambridge University Press), 553

Baudoz, P., Mazoyer, J., Mas, M., Galicher, R., \& Rousset, G. 2012, in Groundbased and Airborn Intrusmentation for Astronomy IV, Proc. Soc. Photo-Opt. Instrum. Eng., 8446

Beuzit, J.-L., Feldt, M., Dohlen, K., et al. 2007, in Proc. Conference In the Spirit of Bernard Lyot: The Direct Detection of Planets and Circumstellar Disks in the 21st Century, ed. P. Kalas (University of California, Berkeley, CA, USA)

Bordé, P. J., \& Traub, W. A. 2006, ApJ, 638

Born, M., \& Wolf, E. 1989, Principles of Optics (Pergamon Press)

Give'on, A., Belikov, R., Shaklan, S., \& Kasdin, J. 2007, Opt. Express, 15

Gonsalvez, R. 1982, Opt. Eng., 21

Gratadour, D., Mugnier, L. M., \& Rouan, D. 2005, A\&A, 443, 357

Guyon, O., Roddier, C., Graves, J., et al. 1999, PASP, 111

Guyon, O., Matsuo, T., \& Angel, R. 2009, ApJ, 693

Kalas, P., Graham, J. R., Chiang, E., et al. 2008, Science, 332

Lagrange, A.-M., Gratadour, D., Chauvin, G., et al. 2009, A\&A, 493, L21

Macintosh, B. A., Graham, J. R., Palmer, D. W., et al. 2008, in Adaptive Optics Systems, Proc. Soc. Photo-Opt. Instrum. Eng., 7015

Marois, C., Macintosh, B., Barman, T., et al. 2008, Science, 322

Mugnier, L. M., Robert, C., Conan, J.-M., Michau, V., \& Salem, S. 2001, J. Opt. Soc. Am. A, 18, 862

Mugnier, L. M., Fusco, T., \& Conan, J.-M. 2004, J. Opt. Soc. Am. A, 21, 1841

Mugnier, L. M., Blanc, A., \& Idier, J. 2006, in Advances in Imaging and Electron Physics, ed. P. Hawkes (Elsevier), 141, 1

Noll, R. J. 1976, J. Opt. Soc. Am., 66, 207

Press, W. H., Teukolsky, S. A., Vetterling, W. T., \& Flannery, B. P. 2007, Numerical Recipes: the art of scientific computing (Cambridge University Press)

Roddier, F., \& Roddier, C. 1997, PASP, 109

Sauvage, J.-F., Fusco, T., Rousset, G., \& Petit, C. 2007, J. Opt. Soc. Am. A, 24, 2334

Sauvage, J.-F., Mugnier, L. M., Rousset, G., \& Fusco, T. 2010, J. Opt. Soc. Am. A, 27, A157

Sauvage, J.-F., Mugnier, L., Paul, B., \& Villecroze, R. 2012, Opt. Lett., 37, 4808

Soummer, R., Aime, C., \& Falloon, P. 2003, A\&A, 397, 1161

Soummer, R., Pueyo, L., Sivaramakrishnan, A., \& Vanderbei, R. 2007, Opt. Express, 15

Thiébaut, E. 2002, in Astronomical Data Analysis II, Proc. Soc. Photo-Opt. Instrum. Eng., 4847, 174

Thiébaut, E., \& Conan, J.-M. 1995, J. Opt. Soc. Am. A, 12, 485

Trauger, J., Give'on, A., Gordon, B., et al. 2010, in Tecniques and Instrumentation for Detection of Exoplanets III, Proc. Soc. Photo-Opt. Instrum. Eng., 6693

Wallace, J. K., Burruss, R. S., Bartos, R. D., et al. 2010, in Adaptive Optics Systems II, Proc. Soc. Photo-Opt. Instrum. Eng., 7736 